\title{
Spatial PAPR Reduction in Symbol-level Precoding for the Multi-beam Satellite Downlink
}

\author{
Danilo Spano*, Maha Alodeh*, Symeon Chatzinotas*, Jens Krause ${ }^{\dagger}$, and Björn Ottersten* \\ ${ }^{*}$ SnT - securityandtrust.lu, University of Luxembourg \\ email: \{danilo.spano, symeon.chatzinotas, bjorn.ottersten\}@uni.lu \\ ${ }^{\dagger}$ SES, Chateau de Betzdorf, Luxembourg, email: jens.krause@ @es.com
}

\begin{abstract}
In this work, a novel symbol-level precoding scheme is proposed, for managing the multi-user interference in the forward downlink channel of a multi-beam satellite system. Besides exploiting the constructive interference effect, the proposed scheme aims at improving the robustness of the transmitted signals to the non-linear distortions of practical satellite systems. This is done by reducing the imbalances between the instantaneous power transmitted by the multiple antennas, which are detrimental in non-linear systems. More specifically, this work proposes a symbol-level precoding scheme performing the minimization of the spatial peak-to-average power ratio, under Quality-of-Service constraints. An iterative algorithm is proposed to solve the related optimization problem. Numerical results are presented to assess the performance of the proposed scheme, which outperforms the state of the art symbol-level precoding techniques in terms of spatial peak-to-average power ratio across the transmitting antennas.
\end{abstract}

\section{INTRODUCTION}

In the current research on satellite communications (SatComs), an effective strategy in order to fulfill the everincreasing throughput requirements relies on multi-beam architectures implementing aggressive frequency reuse schemes. These schemes, based on the utilization of multiple antennas at the transmitter side, require the development of advanced signal processing techniques, able to handle the problem of multi-user interference (MUI) arising in multi-beam systems and deteriorating their performance. In this context, linear precoding (or beamforming) techniques have been a prolific recent area in the recent years, showing to be an effective way to manage the MUI while guaranteeing some specific service requirements [1]-[3].

Precoding techniques can be classified in block-level and symbol-level. Block-level precoding is the conventional precoding strategy, where the knowledge of the channel state information (CSI) is exploited in order to design a precoding weight matrix (or precoder) to be applied to the multiple data streams. Since the precoder depends only on the CSI, it remains constant for a whole block of symbols whose length is related to the coherence time of the channel. Several blocklevel schemes have been proposed in the literature, optimizing different objectives [1]-[5]. On the other hand, symbollevel precoding (SLP) is a novel strategy [6]-[11] where the transmitted signals are designed using the knowledge of both the CSI and the data information (DI), namely the symbols to be delivered to the users. This allows to handle the MUI in a more effective way with respect to the conventional schemes. In particular, SLP has the objective to achieve a constructive interference effect at the receiver side [6], [7], and to exploit it to improve the system performance. Different optimization strategies have been considered in the literature for SLP, including sum power minimization schemes and maxmin fair formulations ${ }^{1}$ [8], [9], [12]. The potential of SLP has been further explored by the authors in [13], [14], where the per-antenna power limitations of the transmitter are accounted.

This paper copes with the problem of symbol-level precoding over non-linear channels, with particular reference to SatComs systems, and takes a step forward with respect to the previous works [13], [14]. The non-linear effects of the satellite channel, introduced by the on-board per-antenna traveling-wave-tube amplifiers (TWTAs), are a well known problem, widely investigated in the literature [15]-[18]. Such effects are even more harmful in multi-beam systems, where the distortion applies independently to each transmitted stream, depending on the instantaneous transmitted power. As a consequence, a high variation between the instantaneous power transmitted by different antennas determines different phase shifts in the amplification stages, and this differential effect is a further source of performance degradation. In order to tackle this problem, this works proposes a SLP scheme aiming at minimizing the power imbalances between the transmit antennas, on a symbol basis, so to increase the robustness to the on-board non-linear TWTAs. More specifically, the symbollevel problem of minimizing the spatial peak-to-average power ratio (SPAPR) ${ }^{2}$, under QoS constraints, is proposed and solved for M-PSK modulations. The novel aspect of the proposed approach with respect to the state of the art [13], [14] is in the fact that the issue of non-linearities is directly addressed by minimizing the SPAPR, instead of considering the per-antenna transmit power as the optimization objective.

The remainder of the paper is organized as follows. In Section II, the system and signals communication model is

\footnotetext{
${ }^{1}$ The max-min fair approach aims to maximize the minimum signal-tointerference-plus-noise ratio (SINR) amongst the users, in order to preserve the fairness of the system.

${ }^{2}$ In the terrestrial communications framework there are a number of works, such as [19], proposing precoding techniques for PAPR reduction. It shall be stressed that therein the PAPR is intended in the temporal dimension, while in this work we address a different problem, i.e., the reduction of the spatial PAPR (SPAPR) in SLP schemes achieving constructive interference.
} 
delineated. In Section III, the problem of SLP for SPAPR minimization is formulated and solved. In Section IV, the proposed approach is validated through simulation results. Finally, in Section V conclusions are drawn.

Notation: $(\cdot)^{T}$ denotes matrix transpose, while $(\cdot)^{\dagger}$ denotes matrix conjugate transpose. $|\cdot|$ and $\angle(\cdot)$ denote the amplitude and the phase, respectively, while $\operatorname{Re}(\cdot)$ and $\operatorname{Im}(\cdot)$ are the real and imaginary parts. Moreover, $\|\cdot\|$ and $\|\cdot\|_{\infty}$ represent the Euclidean norm and the $1_{\infty}$ norm, respectively. Finally, $\circ$ is used to denote the element-wise Hadamard matrix product.

\section{System and Signals Communication Model}

We consider a multi-user (MU) multiple-input single-output (MISO) satellite system, and we denote by $K$ the number of antennas of the on-board transmitter. These antennas allow the generation of $K$ independent beams through a parabolic reflector. Let us also assume that $K$ single-antenna users are served in a specific instant, namely considering one user per beam $^{3}$. The adopted modulation is M-PSK, and a channel vector $\boldsymbol{h}_{j} \in \mathbb{C}^{1 \times K}$ is assumed between the transmitting antennas and the $j$-th user. The received signal at the $j$-th user in the symbol slot $n$ can be written as:

$$
y_{j}[n]=\boldsymbol{h}_{j} \boldsymbol{x}[n]+z_{j}[n],
$$

where $\boldsymbol{x}[n] \in \mathbb{C}^{K \times 1}$ represents the transmitted signal vector from the $K$ transmit antennas, and $z_{j}[n]$ is a complex circular symmetric random variable, modeling the zero mean Additive White Gaussian Noise (AWGN) measured at the $j$-th user's receiving antenna. Without loss of generality, the noise variance is assumed to be 1 .

The introduced communication model can be written in a more compact form as follows:

$$
\boldsymbol{y}[n]=\boldsymbol{H} \boldsymbol{x}[n]+\boldsymbol{z}[n],
$$

where $\boldsymbol{y}[n] \in \mathbb{C}^{K \times 1}$ and $\boldsymbol{z}[n] \in \mathbb{C}^{K \times 1}$ collect the received signals and the AWGN components at all the users, respectively, while $\boldsymbol{H}=\left[\boldsymbol{h}_{1}^{T} \ldots \boldsymbol{h}_{K}^{T}\right]^{T} \in \mathbb{C}^{K \times K}$ represents the system channel matrix. Following the general SLP scheme of [8], the transmitted signal vector $\boldsymbol{x}[n]$ is designed by a precoding module, which takes as input the CSI, i.e., an estimate of $\boldsymbol{H}$, and the DI vector $\boldsymbol{d}[n] \in \mathbb{C}^{K \times 1}$, representing the data symbols to be conveyed to the users. The data symbols, drawn from an M-PSK constellation, are assumed to be uncorrelated and having unit power.

The matrix $\boldsymbol{H}$, modeling the multi-beam satellite channel, is a complex matrix that can be written as follows:

$$
\boldsymbol{H}=\boldsymbol{\Phi} \circ \boldsymbol{B},
$$

where $\boldsymbol{B} \in \mathbb{R}^{K \times K}$ is a matrix modeling the multi-beam satellite radiation pattern, while $\boldsymbol{\Phi} \in \mathbb{C}^{K \times K}$ models the signal phase rotations induced by the the on-board RF chains and by the propagation paths.

\footnotetext{
${ }^{3}$ This is reasonable if the systems resorts to a time-division multiplexing scheme, in order to serve all the users in each beam.
}

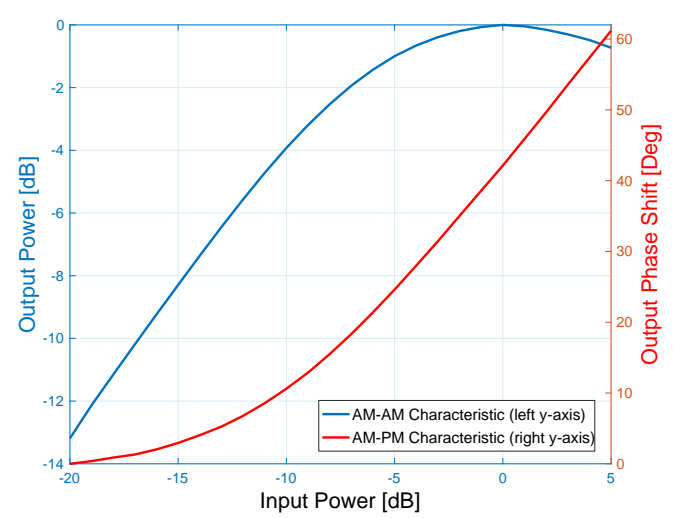

Figure 1: Normalized AM-AM and AM-PM characteristics of the onboard TWTAs (non-linearized model).

As regard to the real matrix $\boldsymbol{B}$, its elements depend on the multi-beam radiation pattern and on the users position. Considering the $j$-th beam and the $k$-th user, the corresponding entry of the matrix $\boldsymbol{B}$ can be calculated resorting to the well accepted method of Bessel functions, thus it will be the square root of the following power gain [20]:

$$
g_{j k}\left(\theta_{j k}\right)=G_{\max }\left(\frac{J_{1}(u)}{2 u}+36 \frac{J_{3}(u)}{u^{3}}\right)^{2},
$$

where $\theta_{j k}$ is the off-axis angle of the user with respect to the boresight of the beam, $u=2.07123 \sin \theta_{j k} / \sin \theta_{3 \mathrm{~dB}}$, with $\theta_{3 \mathrm{~dB}}$ being the one-sided half-power angular beamwidth, $G_{\max }$ is the maximum on-axis power gain of the antenna, and $J_{1}$, $J_{3}$ are the Bessel functions of the first kind, of order one and three respectively.

Regarding the phase rotations, they are assumed independent for each antenna-user pair, so as to take into account the different propagation paths between the transmitter and the users, as well as the different on-board RF chains. Accordingly, the generic element of the matrix $\boldsymbol{\Phi}$ in (3) is modeled as $[\boldsymbol{\Phi}]_{j k}=e^{i \phi_{j k}}$, where $\phi_{j k}$ are independent random variables uniformly distributed in $[0,2 \pi) \forall j, k=1, \ldots, K$.

\section{A. Non-linearities of the Satellite Channel}

As anticipated, it should be taken into account how the system model introduced in (2) is actually degraded by the nonlinear effects introduced by the on-board per-antenna TWTAs. Different models describing the amplitude and phase distortion introduced by the amplifiers are provided in [17], [18]. We take as a reference the common non-linearized TWTA model, whose normalized amplitude-to-amplitude (AM-AM) and amplitude-to-phase (AM-PM) characteristics are shown in Fig. 1. The characteristics clearly show the saturation effect and the introduced phase shifts.

Since the on-board TWTAs need to be operated as close as possible to saturation, to efficiently exploit the scarce available power, it becomes very important to have some control over the power variation of the precoded waveforms, in order to avoid high power peaks. Moreover, in systems using 
separate per-antenna TWTAs, a different phase distortion is induced over the various data streams, because of the different instantaneous power carried out by the symbols of each stream. This additional issue, which we refer to as differential phase shift, is particularly relevant when precoding is applied, since the power imbalances between the transmitting antennas are usually not controlled. As a consequence, a reduced power variation of the precoded waveforms is needed also in the spatial dimension, i.e., between the different antennas. In this direction, the SLP scheme proposed in the following section aims at minimizing the SPAPR in the transmitted signal vector $\boldsymbol{x}[n]$, so as to make it more robust to the non-linear channel, and specifically to the differential phase shift.

\section{Spatial PeAK-To-AVERAge Power Ratio MINIMIZATION}

In line with the general framework of SLP, the goal is to exploit the knowledge of the CSI and of the DI in order to design the transmitted vector $\boldsymbol{x}$ (to ease the notation, hereafter the time index $n$ is omitted), assuring that the received signal lies in the detection region of the desired symbol, for each user. In other words, we target a constructive interference effect, as in [8]. The novel aspect of the proposed scheme is in the optimization objective, which is the SPAPR amongst the transmitting antennas defined as $\frac{\|\boldsymbol{x}\|_{\infty}^{2}}{\|\boldsymbol{x}\|^{2} / K}$.

More specifically, the proposed SLP approach performs the minimization of the SPAPR under QoS constraints. The related optimization problem, referred to as SPAPR-Min, can be formulated as a non-linear fractional programming as follows:

$$
\begin{aligned}
\boldsymbol{x}(\boldsymbol{d}, \boldsymbol{H}, \boldsymbol{\gamma})= & \underset{\boldsymbol{x}}{\arg \min _{\boldsymbol{x}}} \frac{\|\boldsymbol{x}\|_{\infty}^{2}}{\|\boldsymbol{x}\|^{2}} \\
& \text { subject to } \\
\mathcal{C} 1: & \left|\boldsymbol{h}_{j} \boldsymbol{x}\right|^{2} \geq \gamma_{j}, j=1, \ldots, K, \\
\mathcal{C} 2: \quad & \angle \boldsymbol{h}_{j} \boldsymbol{x}=\angle d_{j}, j=1, \ldots, K,
\end{aligned}
$$

where $\gamma_{j}$ is the target signal-to-interference-plus-noise ratio (SINR) that should be granted for the $j$-th user, and $\gamma=$ $\left[\gamma_{1} \ldots \gamma_{K}\right]^{T} \in \mathbb{C}^{K \times 1}$ stacks the target SINR for all the users. The set of constraints $\mathcal{C} 1$ represents a QoS constraint for each user, while the set of constraints $\mathcal{C} 2$ represents the constructive interference condition, guaranteeing that each user receives the desired data symbol with the correct phase.

Similarly to [13], the problem (5) can be reformulated as follows:

$$
\begin{aligned}
\boldsymbol{x}(\boldsymbol{d}, \boldsymbol{H}, \boldsymbol{\gamma})= & \arg \min _{\boldsymbol{x}} \frac{\|\boldsymbol{x}\|_{\infty}^{2}}{\|\boldsymbol{x}\|^{2}} \\
& \operatorname{subject} \text { to } \\
\mathcal{C} 1: \quad & \operatorname{Re}\left(d_{j}\right) \operatorname{Re}\left(\boldsymbol{h}_{j} \boldsymbol{x}\right) \geq \sqrt{\gamma_{j}} \operatorname{Re}^{2}\left(d_{j}\right), \\
& j=1, \ldots, K, \\
\mathcal{C} 2: \quad \operatorname{Im}\left(d_{j}\right) \operatorname{Im}\left(\boldsymbol{h}_{j} \boldsymbol{x}\right) \geq \sqrt{\gamma_{j}} \operatorname{Im}^{2}\left(d_{j}\right), & j=1, \ldots, K, \\
\mathcal{C} 3: & \frac{\operatorname{Im}\left(\boldsymbol{h}_{j} \boldsymbol{x}\right)}{\operatorname{Re}\left(\boldsymbol{h}_{j} \boldsymbol{x}\right)}=\alpha_{j}, j=1, \ldots, K,
\end{aligned}
$$

To be presented in IEEE SPAWC 2017 where $\alpha_{j}=\tan \left(\angle d_{j}\right) \forall j=1, \ldots, K$. In this formulation, the constraints are referred to the in-phase and quadrature components of the received signals. Furthermore, by taking into account that $\operatorname{Re}\left(\boldsymbol{h}_{j} \boldsymbol{x}\right)=\frac{\boldsymbol{h}_{j} \boldsymbol{x}+\boldsymbol{x}^{\dagger} \boldsymbol{h}_{j}^{\dagger}}{2}$ and $\operatorname{Im}\left(\boldsymbol{h}_{j} \boldsymbol{x}\right)=$ $\frac{\boldsymbol{h}_{j} \boldsymbol{x}-\boldsymbol{x}^{\dagger} \boldsymbol{h}_{j}^{\dagger}}{2 i}$, the problem can be straightforwardly expressed as:

$$
\begin{aligned}
& \boldsymbol{x}(\boldsymbol{d}, \boldsymbol{H}, \boldsymbol{\gamma})= \arg \min _{\boldsymbol{x}} \frac{\|\boldsymbol{x}\|_{\infty}^{2}}{\|\boldsymbol{x}\|^{2}} \\
& \text { subject to } \\
& \mathcal{C} 1: \quad \operatorname{Re}\left(d_{j}\right) \frac{\boldsymbol{h}_{j} \boldsymbol{x}+\boldsymbol{x}^{\dagger} \boldsymbol{h}_{j}^{\dagger}}{2} \geq \sqrt{\gamma_{j}} \operatorname{Re}^{2}\left(d_{j}\right), \\
& j=1, \ldots, K, \\
& \mathcal{C} 2: \quad \operatorname{Im}\left(d_{j}\right) \frac{\boldsymbol{h}_{j} \boldsymbol{x}-\boldsymbol{x}^{\dagger} \boldsymbol{h}_{j}^{\dagger}}{2 i} \geq \sqrt{\gamma_{j}} \operatorname{Im}^{2}\left(d_{j}\right), \\
& j=1, \ldots, K, \\
& \mathcal{C} 3: \quad\left(i \alpha_{j}-1\right) \boldsymbol{h}_{j} \boldsymbol{x}+\left(i \alpha_{j}+1\right) \boldsymbol{x}^{\dagger} \boldsymbol{h}_{j}^{\dagger}=0, \\
& j=1, \ldots, K .
\end{aligned}
$$

Although the constraints have been reformulated in a tractable form, the problem (7) is still challenging because of the non-linear fractional objective function. In order to solve it, we can resort to an approach based on parametric programming [21], which allows to tackle fractional programs by iteratively solving a sequence of convex problems which converges to the global solution. Nevertheless, the method of [21] requires the objective function of the fractional program to be a ratio of a convex function over a concave one. Since this is not the case of the problem (7), we first need to approximate the function $\|\boldsymbol{x}\|^{2}$ as an affine function around a generic point $\boldsymbol{z} \in \mathbb{C}^{K \times 1}$, as:

$$
\begin{aligned}
(\boldsymbol{x}-\boldsymbol{z})^{\dagger}(\boldsymbol{x}-\boldsymbol{z})= & \boldsymbol{x}^{\dagger} \boldsymbol{x}-2 \operatorname{Re}\left(\boldsymbol{z}^{\dagger} \boldsymbol{x}\right)+\boldsymbol{z}^{\dagger} \boldsymbol{z} \geq 0 \\
& \boldsymbol{x}^{\dagger} \boldsymbol{x} \geq 2 \operatorname{Re}\left(\boldsymbol{z}^{\dagger} \boldsymbol{x}\right)-\boldsymbol{z}^{\dagger} \boldsymbol{z} \\
& \boldsymbol{x}^{\dagger} \boldsymbol{x} \approx 2 \operatorname{Re}\left(\boldsymbol{z}^{\dagger} \boldsymbol{x}\right)-\boldsymbol{z}^{\dagger} \boldsymbol{z}
\end{aligned}
$$

With this affine approximation (which can be considered in the framework of successive convex approximation - SCA [22]), we can apply the parametric programming approach of [21] to the problem at hand. To this aim, we shall define the optimization function $F(\lambda)=\min _{\boldsymbol{x} \in \mathcal{S}}\left\{\|\boldsymbol{x}\|_{\infty}^{2}-\lambda\left(2 \operatorname{Re}\left(\boldsymbol{z}_{k}^{\dagger} \boldsymbol{x}\right)-\boldsymbol{z}_{k}^{\dagger} \boldsymbol{z}_{k}\right)\right\}$, where $\mathcal{S}$ represents the sets of constraints $\mathcal{C}_{1}, \mathcal{C}_{2}, \mathcal{C}_{3}$, and $\lambda$ is an auxiliary variable. The resulting problem can be written as in (9). Ultimately, the problem (7) can be solved using the parametric formulation given in (9), through the iterative algorithm summarized in Table I. It should be highlighted that the iterative approach involves the update of both the parameter $\lambda$ and the SCA point $\boldsymbol{z}$. 
1) Initialization: Set $\epsilon, k=0, \lambda=0$ in (9), which results in solving $F(0)$.

2) Evaluate $\lambda_{0}=\frac{\|\boldsymbol{x}\|_{\infty}^{2}}{\|\boldsymbol{x}\|^{2}}, \boldsymbol{z}_{0}=\boldsymbol{x}$

3) Solve the following optimization:

$$
\begin{aligned}
\boldsymbol{x}= & \underset{\boldsymbol{x}}{\arg \min _{\boldsymbol{x}}}\|\boldsymbol{x}\|_{\infty}^{2}-\lambda_{k}\left(2 \operatorname{Re}\left(\boldsymbol{z}_{k}^{\dagger} \boldsymbol{x}\right)-\boldsymbol{z}_{k}^{\dagger} \boldsymbol{z}_{k}\right) \\
& \text { subject to } \\
\mathcal{C} 1: \quad & \operatorname{Re}\left(d_{j}\right) \frac{\boldsymbol{h}_{j} \boldsymbol{x}+\boldsymbol{x}^{\dagger} \boldsymbol{h}_{j}^{\dagger}}{2} \geq \sqrt{\gamma_{j}} \operatorname{Re}^{2}\left(d_{j}\right), \\
& j=1, \ldots, K, \\
\mathcal{C} 2: \quad & \operatorname{Im}\left(d_{j}\right) \frac{\boldsymbol{h}_{j} \boldsymbol{x}-\boldsymbol{x}^{\dagger} \boldsymbol{h}_{j}^{\dagger}}{2 i} \geq \sqrt{\gamma_{j}} \operatorname{Im}^{2}\left(d_{j}\right), \\
& j=1, \ldots, K, \\
\mathcal{C} 3: \quad & \left(i \alpha_{j}-1\right) \boldsymbol{h}_{j} \boldsymbol{x}+\left(i \alpha_{j}+1\right) \boldsymbol{x}^{\dagger} \boldsymbol{h}_{j}^{\dagger}=0, \\
& j=1, \ldots, K .
\end{aligned}
$$

4) Evaluate $\left|F\left(\lambda_{k}\right)\right|$ and $\left\|\boldsymbol{x}-\boldsymbol{z}_{k}\right\|$; if $F\left(\lambda_{k}\right) \geq \epsilon$ or $\left\|\boldsymbol{x}-\boldsymbol{z}_{k}\right\| \geq \epsilon$ go to step 5 .

5) Set $\lambda_{k+1}=\frac{\|\boldsymbol{x}\|_{\infty}^{2}}{\|\boldsymbol{x}\|^{2}}, \boldsymbol{z}_{k+1}=\boldsymbol{x}, k=k+1$, go to step 3 .

$$
\begin{aligned}
\boldsymbol{x}(\boldsymbol{d}, \boldsymbol{H}, \boldsymbol{\gamma})= & \underset{\boldsymbol{x}, \lambda}{\arg \min _{\boldsymbol{x}}}\|\boldsymbol{x}\|_{\infty}^{2}-\lambda\left(2 \operatorname{Re}\left(\boldsymbol{z}^{\dagger} \boldsymbol{x}\right)-\boldsymbol{z}^{\dagger} \boldsymbol{z}\right) \\
& \text { subject to } \\
\mathcal{C} 1: \quad & \operatorname{Re}\left(d_{j}\right) \frac{\boldsymbol{h}_{j} \boldsymbol{x}+\boldsymbol{x}^{\dagger} \boldsymbol{h}_{j}^{\dagger}}{2} \geq \sqrt{\gamma_{j}} \operatorname{Re}^{2}\left(d_{j}\right), \\
& j=1, \ldots, K, \\
\mathcal{C} 2: \quad & \operatorname{Im}\left(d_{j}\right) \frac{\boldsymbol{h}_{j} \boldsymbol{x}-\boldsymbol{x}^{\dagger} \boldsymbol{h}_{j}^{\dagger}}{2 i} \geq \sqrt{\gamma_{j}} \operatorname{Im}^{2}\left(d_{j}\right), \\
& j=1, \ldots, K \\
\mathcal{C} 3: \quad & \left(i \alpha_{j}-1\right) \boldsymbol{h}_{j} \boldsymbol{x}+\left(i \alpha_{j}+1\right) \boldsymbol{x}^{\dagger} \boldsymbol{h}_{j}^{\dagger}=0, \\
& j=1, \ldots, K .
\end{aligned}
$$

The parametric problem (9) can be solved using the standard convex optimization tools. Both the SCA approach [22] and the parametric programming [21] have been proven convergent. Moreover, a numerical analysis has evidenced a fast convergence of the proposed joint algorithm.

\section{NumericAl Results}

In this section some numerical results are presented, in order to validate the proposed SPAPR-Min scheme. The presented results are obtained assuming a 7-beam satellite channel based on the radiation pattern described by (4), with a maximum power gain $G_{\max }=10 \mathrm{~dB}$. The position of each user is fixed in the center of the respective beam. Moreover, the considered modulation is 8-PSK. Before presenting the numerical results, let us introduce the considered performance metrics. The main metric considered to evaluate the robustness of the proposed technique to the non-linear impairments is the SPAPR, defined as SPAPR $=\frac{\|x\|_{\infty}^{2}}{\|\boldsymbol{x}\|^{2} / K}$. Moreover, some power metrics are also

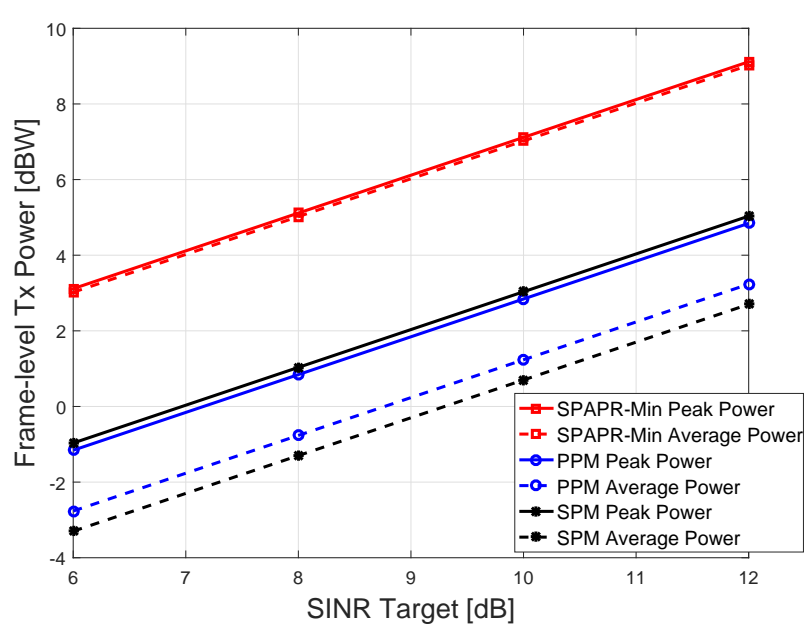

Figure 2: Frame-level peak and average power, in $\mathrm{dBW}$, versus target SINR, in $\mathrm{dB}$.

evaluated. The symbol-level average power transmitted by the antennas is $P_{\mathrm{av}}=\frac{\|\boldsymbol{x}\|^{2}}{K}$. Furthermore, the symbol-level peak power amongst the antennas is defined as $P_{\text {peak }}=\|\boldsymbol{x}\|_{\infty}^{2}$. By taking an average of the introduced quantities (symbol-level SPAPR and power metrics) over a large number of symbol slots, we obtain the related frame-level values, which are used in the results presented hereafter.

We consider as benchmarks two different SLP schemes. One is the SLP approach proposed in [8], performing a sum power minimization (SPM) with QoS constraints. The other benchmark is the technique proposed in [13], where the peak power minimization (PPM) amongst the transmitting antennas is performed at a symbol level, under QoS constraints.

Fig. 2 shows the frame-level peak power and average power, in $\mathrm{dBW}$, for the proposed techniques and the benchmarks, as a function of the target SINR, in dB. The attained SPAPR for the proposed approach is $0.01 \mathrm{~dB}$, while the corresponding values for the PPM and the SPM approaches are $1.6 \mathrm{~dB}$ and $2.34 \mathrm{~dB}$, respectively. Remarkably, it is visible how the proposed scheme not only outperforms the benchmarks in terms of SPAPR, but it is also very close to the theoretical lower bound for such metric, which is $0 \mathrm{~dB}$. This means that, for each symbol slot, the power imbalances between the different antennas are completely removed, with a peak power coinciding with the average power. Therefore, the transmitted waveforms do not suffer any differential phase shift. On the other hand, there is a considerable increase in the power absolute values with respect to the SPM and PPM schemes. Overall, the results show how the proposed SPAPRMin scheme allows to remarkably improve the spatial dynamic of the transmitted signals, at the expense of a higher required power. This can be explained considering how, differently than the PPM and SPM schemes, the SPAPR-Min optimization problem (5) does not directly minimize the transmit power.

To give further insights on the SPAPR and power performance of the proposed scheme, Fig. 3 displays the per-antenna 


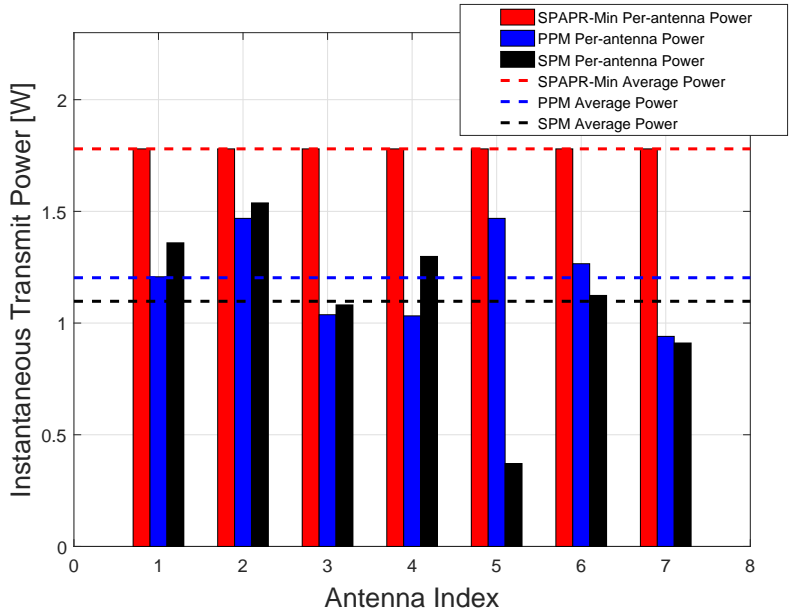

Figure 3: Per-antenna instantaneous power utilization, in $\mathrm{W}$, for a specific symbol slot.

instantaneous power utilization, in linear scale, for a specific symbol slot. Moreover, also the average power is shown for the three techniques. The target SINR is fixed to $8 \mathrm{~dB}$. This representation clearly shows how the SPAPR-Min technique sacrifices some power efficiency in order to have a much more balanced distribution among the antennas, thus being more robust with respect to the non-linear effects.

As a final remark, it should be mentioned that the average number of iterations needed to reach the convergence is 4.5 , independently on the target SINR.

\section{CONCLUSIONS}

In this paper, a novel symbol-level precoding technique has been proposed, with the objective of reducing the instantaneous power imbalances between the multiple transmitting antennas. This objective is particularly relevant in systems using separate per-antenna power amplifiers introducing nonlinear effects on the transmitted waveforms, which is a typical case in SatComs. More specifically, the proposed approach performs the minimization of the spatial peak-to-average power ratio, under Quality-of-Service constraints. This design allows to reduce the degradation due to the satellite channel non-linearities, in particular with respect to the differential phase distortion. Numerical results have been presented to assess the performance of the technique with respect to the state of the art, showing how a unit SPAPR can be attained at the expense of a higher power consumption.

\section{ACKNOWLEDGMENT}

This work was partially supported by H2020 project SANSA, and by Fond National de la Recherche Luxembourg (FNR), under the projects SeMiGod, SATSENT, and BroadSat (AFR project).

\section{REFERENCES}

[1] M. Bengtsson and B. Ottersten, "Optimal and suboptimal transmit beamforming," in Handbook of Antennas in Wireless Communications. CRC Press, 2001, pp. 18-1-18-33.
[2] M. Schubert and H. Boche, "Solution of the multiuser downlink beamforming problem with individual SINR constraints," IEEE Transactions on Vehicular Technology, vol. 53, no. 1, pp. 18-28, Jan 2004.

[3] M. Bengtsson and B. Ottersten, "Optimal downlink beamforming using semidefinite optimization," in Proc. of Annual Allert. Conf. on Commun. Control and Computing, vol. 37. Citeseer, 1999, pp. 987-996.

[4] W. Yu and T. Lan, "Transmitter optimization for the multi-antenna downlink with per-antenna power constraints," IEEE Transactions on Signal Processing, vol. 55, no. 6, pp. 2646-2660, June 2007.

[5] G. Zheng, S. Chatzinotas, and B. Ottersten, "Generic optimization of linear precoding in multibeam satellite systems," IEEE Transactions on Wireless Communications, vol. 11, no. 6, pp. 2308-2320, June 2012.

[6] C. Masouros and E. Alsusa, "Dynamic linear precoding for the exploitation of known interference in MIMO broadcast systems," IEEE Transactions on Wireless Communications, vol. 8, no. 3, pp. 1396-1404, March 2009.

[7] C. Masouros, "Correlation rotation linear precoding for MIMO broadcast communications," IEEE Transactions on Signal Processing, vol. 59, no. 1, pp. $252-262$, Jan. 2011.

[8] M. Alodeh, S. Chatzinotas, and B. Ottersten, "Constructive multiuser interference in symbol level precoding for the MISO downlink channel," IEEE Transactions on Signal Processing,, vol. 63, no. 9, pp. 2239-2252, May 2015.

[9] M. Alodeh, S. Chatzinotas, and B. Ottersten, "Energy-efficient symbollevel precoding in multiuser MISO based on relaxed detection region," IEEE Transactions on Wireless Communications, vol. 15, no. 5, pp. 3755-3767, May 2016.

[10] C. Masouros and G. Zheng, "Exploiting known interference as green signal power for downlink beamforming optimization," IEEE Transactions on Signal Processing, vol. 63, no. 14, pp. 3628-3640, July 2015.

[11] A. Kalantari, M. Soltanalian, S. Maleki, S. Chatzinotas, and B. Ottersten, "Directional modulation via symbol-level precoding: A way to enhance security,' IEEE Journal of Selected Topics in Signal Processing, vol. 10, no. 8, pp. 1478-1493, Dec 2016.

[12] M. Alodeh, S. Chatzinotas, and B. Ottersten, "Constructive interference through symbol level precoding for multi-level modulation," in 2015 IEEE Global Communications Conference (GLOBECOM), Dec 2015, pp. $1-6$.

[13] D. Spano, M. Alodeh, S. Chatzinotas, and B. Ottersten, "Per-antenna power minimization in symbol-level precoding," in 2016 IEEE Global Communications Conference (GLOBECOM), Dec 2016, pp. 1-6.

[14] D. Spano, S. Chatzinotas, J. Krause, and B. Ottersten, "Symbollevel precoding with per-antenna power constraints for the multi-beam satellite downlink," in 2016 8th Advanced Satellite Multimedia Systems Conference and the 14th Signal Processing for Space Communications Workshop (ASMS/SPSC), Sept 2016, pp. 1-8.

[15] E. Casini, R. D. Gaudenzi, and A. Ginesi, "DVB-S2 modem algorithms design and performance over typical satellite channels," International Journal of Sat. Comm. and Netw., vol. 22, no. 3, pp. 281-318, 2004.

[16] D. Spano, D. Christopoulos, S. Andrenacci, S. Chatzinotas, J. Krause, and B. Ottersten, "Total degradation analysis of precoded signals onto non-linear satellite channels," in 21st $\mathrm{Ka}$ and Broadband Communications Conference, Oct 2015.

[17] ETSI EN 302 307-1, "Digital video broadcasting (DVB); second generation framing structure, channel coding and modulation systems for broadcasting, interactive services, news gathering and other broadband satellite applications; part 1: DVB-S2."

[18] ETSI EN 302 307-2, "Digital video broadcasting (DVB); second generation framing structure, channel coding and modulation systems for broadcasting, interactive services, news gathering and other broadband satellite applications; part 2: DVB-S2 extensions (DVB-S2X)."

[19] C. Studer and E. G. Larsson, "Par-aware large-scale multi-user MIMOOFDM downlink," IEEE Journal on Selected Areas in Communications, vol. 31, no. 2, pp. 303-313, February 2013.

[20] M. Diaz, N. Courville, C. Mosquera, G. Liva, and G. Corazza, "Nonlinear interference mitigation for broadband multimedia satellite systems," in Proc. Int. Work. Sat. Space Commun. (IWSSC), Sept. 2007, pp. 61-65.

[21] W. Dinkelbach, "On nonlinear fractional programming," Management Science, vol. 13, no. 7, pp. 492-498, March 1967.

[22] A. Beck, A. Ben-Tal, and L. Tetruashvili, "A sequential parametric convex approximation method with applications to nonconvex truss topology design problems," Journal of Global Optimization, vol. 47, no. 01, pp. 29-51, 2010. 